\title{
Which leg immobilized patient gains the most from Intermittent Pneumatic Compression in DVT-prevention?
}

\author{
R. Juthberg', G. Edman ${ }^{2,4}$, P.W. Ackermann ${ }^{1,3}$ \\ 1 Integrative Orthopedic Laboratory, Department of Molecular Medicine and Surgery, Karolinska Institute, \\ Stockholm, Sweden. \\ 2 Department of Psychiatry, Tiohundra AB, Norrtälje, Sweden. \\ ${ }^{3}$ Department of Orthopedic Surgery, Karolinska University Hospital, Stockholm, Sweden. \\ ${ }^{4}$ Department of Clinical Sciences, Danderyd Hospital, Karolinska Institutet, Stockholm, Sweden.
}

\author{
CORRESPONDING AUTHOR: \\ Robin Juthberg \\ Integrative Orthopedic Laboratory \\ Department of Molecular Medicine and \\ Surgery \\ Karolinska Institute \\ Stockholm, Sweden \\ E-mail: Robin.Juthberg@outlook.com \\ Phone: +46735724462
}

DOI:

10.32098/mltj.02.2019.18

LEVEL OF EVIDENCE:

$2 \mathrm{~B}$

\begin{abstract}
SUMMARY
Background and method. Following Achilles tendon rupture (ATR)-surgery, patients using intermittent pneumatic compression (IPC) of the calf, compared to a plaster casted control-group (CTRL), exhibit reduced risk of deep venous thrombosis (DVT). Based on data from a randomized controlled trial (RCT), we investigated which dichotomized subgroups (based on age, sex, BMI and time to surgery (TTS)) within the IPC- $(n=66)$ and CTRL-group $(n=70)$ that were most likely to sustain a DVT, and which patients that had most benefit from postoperative IPC. Results. Age $\geq 39 y$ (OR 6.06, p<0.001) and TTS $>66 \mathrm{~h}$ (OR 2.3, $\mathrm{p}=0.031$ ) significantly increased the risk of DVT, but not sex or BMI. IPC-treatment significantly reduced the DVT-risk (OR 0.46, $\mathrm{p}=0.042$ ), with more pronounced effect when considering age and TTS (OR 0.40, $\mathrm{p}=0.032$ ). Although non-significant, females (OR 1.44, $\mathrm{p}=0.78$ ) and patients with low BMI (OR 1.16, $\mathrm{p}=0.84$ ) seemed to exhibit less DVT-preventive effect of IPC. Conclusion. Patients with age $>38 y$ and TTS $>66 \mathrm{~h}$ are at increased DVT-risk during post-operative leg immobilization, while adjuvant IPC shows good DVT-preventive effects among most patients, especially in those with high DVT-risk. Further trials are however warranted, to examine the low DVT-preventive effects of IPC discovered among females and patients with low BMI.
\end{abstract}

KEY WORDS

Achilles tendon rupture; deep vein thrombosis; intermittent pneumatic compression

\section{INTRODUCTION}

Injury to the lower limb, such as Achilles tendon rupture (ATR), is most often followed by a period of leg immobilization which is associated with an increased risk to sustain a deep vein thrombosis (DVT) (1). Except the risk of clot propagation to become pulmonary emboli, non-symptomatic DVTs have also been shown to affect patient outcome after ATR (2). Chemical DVT-prophylaxis with low molecular weight heparin has, however, shown little or no DVT-preventive effect $(3,4)$, presumably due to poor blood circulation and low drug concentration in the immobilized limb. Mechanical DVT-prophylaxis with intermittent pneumatic compression (IPC) is an evidence-based therapy in hospitalized patients. Recently IPC was also demonstrated effective in outpatients to reduce the rate of DVT during post-operative leg immobilization after ATR (5). To suggest guidelines for the use of IPC in leg immobilized outpatients, however, the patients with the highest risk of DVT should be identified, and the efficacy of IPC on different subgroups of patients should be analyzed.

The purpose of this study was hence to examine the risk of DVT in subgroups of leg immobilized ATR patients, and to examine if there are subgroups that are more or less likely to obtain a DVT-preventive effect from postoperative treatment with adjuvant IPC beneath an orthosis, as compared to standard immobilization (CTRL) in a plaster cast. The original RCT on which this subgroup-analysis was based, showed a significant benefit from addition of IPC over CTRL (5). 
In this study we report that there not only can be differences between subgroups regarding DVT-incidence, but also differences regarding the DVT-preventive effect of IPC.

\section{METHODS}

\section{Ethical approval}

The original RCT on which this subgroup-analysis was based was performed in accordance with the M.T.J.L. ethical guidelines (6). The original RCT was registered with the United States National Institutes of Health (trial number NCT01317160) and was approved by the Regional Ethical Review Committee in Stockholm, Sweden.

\section{Study design}

Prospectively collected data from a double-blinded randomized controlled trial were used to perform retrospective subgroup-analyses. Subgroups within the IPC- $(\mathrm{n}=66)$ and CTRL-group ( $\mathrm{n}=70$ ) were created by dichotomization of patient characteristic-variables.

\section{Participants and setting}

Between March 2011 and June 2013, 389 patients with an acute Achilles tendon rupture were screened for eligibility at a major hospital in Stockholm, Sweden. Patients with age ranging from 18 to 75 years, who had sustained an acute unilateral Achilles tendon rupture and who had undergone surgery within six days of injury (144 hours, two patients without reported time from injury to surgery) were eligible for inclusion. Exclusion criteria were inability to give informed consent; renal failure; heart failure with pitting edema; thrombophlebitis; thromboembolic event during the previous three months; other surgery during the previous month; anticoagulation treatment (including high dose aspirin); planned follow-up at another hospital; malignancy; hemophilia and pregnancy. Among the 389 patients screened for eligibility, 150 patients (126 men, 24 women) were initially enrolled into the study and thereafter randomized to one of two different post-operative interventions; either conservative treatment with standard plaster cast leg-immobilization (CTRL), or adjuvant intermittent pneumatic compression (IPC) of the calf under an orthosis (Aircast XP Walker, DJO LLC, Vista, California). Randomization was performed using consecutively-numbered sealed envelopes produced by an independent specialist, which were opened shortly after completion of the ATR-surgery.

In the plaster CTRL-group, one patient had been erroneously included despite being under 18 years of age, and in the IPC-group one patient had been erroneously included despite receiving treatment for a thromboembolic event. Both patients were subsequently excluded from the study immediately after randomization. Three patients randomized to IPC-treatment and four patients randomized to plaster cast-treatment chose to terminate their participation in the study prior to the 2-week follow up. Additionally, in the IPC-group, a minimum of 35 hours of IPC-treatment was required to be considered per protocol, and because of this, five patients were excluded from the study due to not reaching the minimum per protocol IPC-time. After exclusion as described above, 66 patients in the IPC-group and 70 patients in the control group completed the study (Figure 1).

\section{Patient demographics}

ATR is much more common among males compared to females. This was reflected in the fact that the overall number of males (115) vs females (21) was skewed in this study. The proportion of females was however not significantly different between the IPC- and CTRL-group. Age, BMI and TTS were normally distributed across the whole patient sample and also non-significantly different between the postoperative treatment alternatives, as described in Table I.

\section{Surgical procedure}

Prior to surgery each patient received $20 \mathrm{ml}$ of local anesthetic (Marcaine $5 \mathrm{mg} / \mathrm{ml}$ with adrenaline) in proximity to the Achilles tendon where after a longitudinal $5-10 \mathrm{~cm}$ dorsomedial skin incision was made in the lower leg on the affected side followed by an incision in the midline of the paratenon. The Achilles tendon stumps were then sutured together end to end with two 1-0 polydioxanone sutures (PDS II) using a modified Kessler technique and the paratenon and fascia cruris were sutured separately using 3-0 Vicryl. Finally, the skin was closed using 3-0 Ethilon sutures. All sutures were supplied by Ethicon, Somerville, NJ, USA.

\section{Post-operative treatment protocol}

Patients in the IPC-group were instructed to apply the intermittent pneumatic compression therapy bilaterally during the time they were sedentary, i.e. sitting or lying in bed sleeping, six hours at a minimum daily, for two weeks following ATR-surgery. The IPC-device (Venaflow ${ }^{\circledR}$ Elite, DJO LLC, Vista, CA, USA) was applied under an orthosis with three wedges (Aircast ${ }^{\circledR}$ XP WalkerTM, DJO LLC, Vista, CA, USA) and the patients were allowed to bear as much weight as they could tolerate. The IPC-device works 


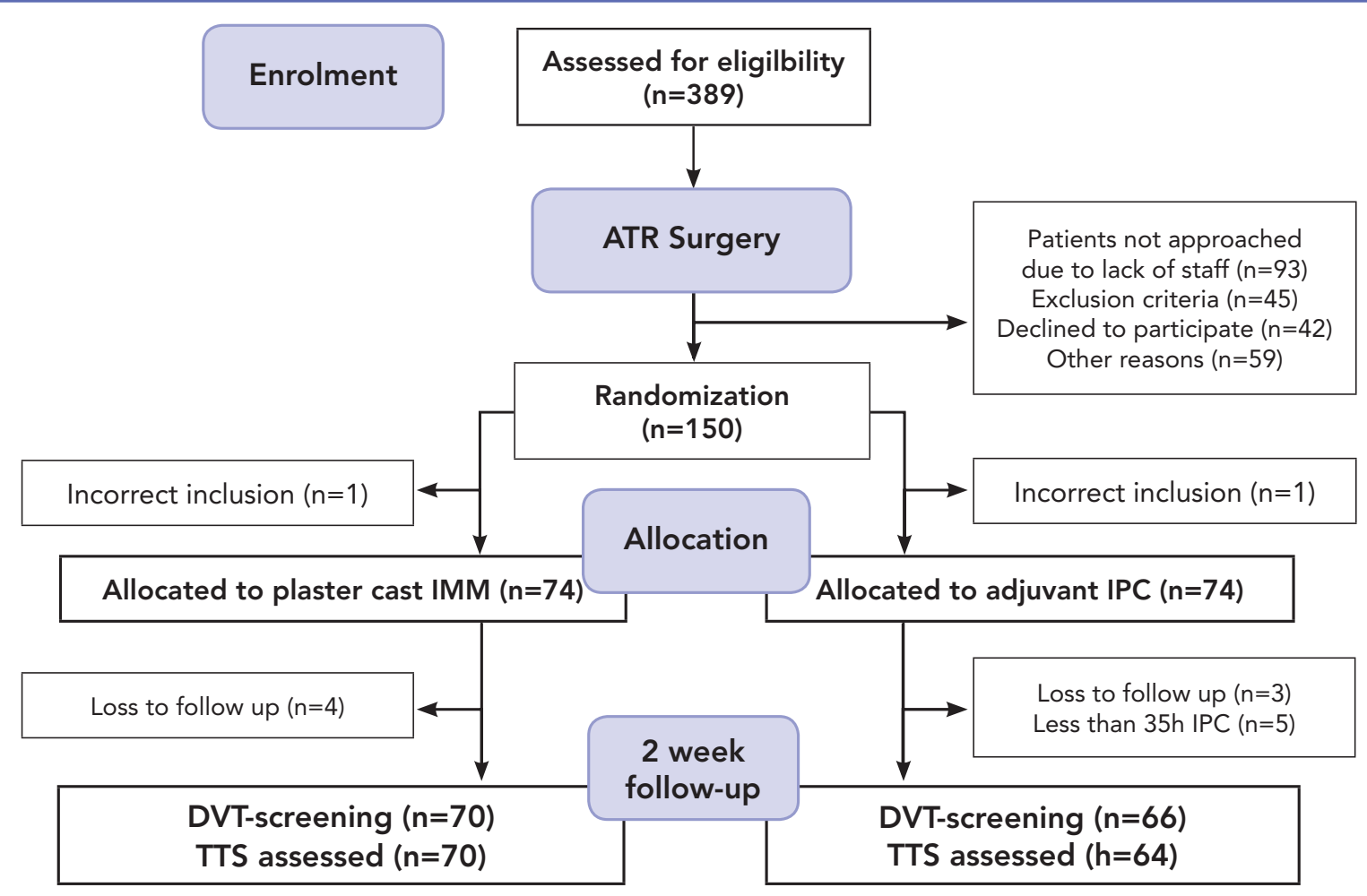

Abbreviations: ATR - Achilles Tendon Rupture, CTRL - Control group with immobilization in plaster cast - Intermittent Pneumatic Compression, DVT - Deep Venous Thrombosis, TTS - Time to Surgery

Figure 1. Patient flow chart

in a cyclic manner where each cycle takes one minute. As the IPC-device cycles, the distal chamber inflates to $73 \mathrm{~mm}$ $\mathrm{Hg}$ over a 0.5 -second period. During the last 0.2 second of this period, the proximal chamber inflates to $63 \mathrm{mmHg}$ and then settles at $45 \mathrm{~mm} \mathrm{Hg}$. After a total of six seconds of inflation, both chambers deflate and remain deflated

Table I. Patient demographics.

\begin{tabular}{|c|c|c|c|}
\hline Variable & IPC $(n=66)$ & CTRL $(n=70)$ & p-value \\
\hline \multicolumn{4}{|l|}{ Sex } \\
\hline Male n (\%) & $58(87.9)$ & $57(81.4)$ & 0.30 \\
\hline Female n $(\%)$ & $8(12.1)$ & $13(18.6)$ & \\
\hline \multicolumn{4}{|l|}{ Age } \\
\hline Mean, years & 40.8 & 39.9 & 0.53 \\
\hline $95 \%$ confidence & $38.9-42.8$ & $37.6-42.2$ & \\
\hline \multicolumn{4}{|l|}{ BMI } \\
\hline Mean, $\mathrm{kg} / \mathrm{cm}^{2}$ & 27.0 & 26.7 & 0.61 \\
\hline $95 \%$ confidence & 26.1-27.9 & $25.9-27.5$ & \\
\hline \multicolumn{4}{|l|}{ TTS } \\
\hline Mean h:m & $63: 55$ & $62: 34$ & 0.76 \\
\hline $95 \%$ confidence & 57.30-70:20 & $56: 40-68: 28$ & \\
\hline
\end{tabular}

Abbreviations: CTRL - Control group with immobilization in plaster cast, IPC - group with Intermittent Pneumatic Compression, BMI - Body Mass Index, TTS - Time to Surgery 
until the next cycle starts. This cycle is then repeated for as long as the IPC-device is being used. The total time of IPC-use was self-registered by the patient as well as by the IPC-device. Since there were individual cases where only the patient, or the IPC-device was able to register the total time of IPC, only the longest registered IPC-time was used for each case in the statistical analysis. Data for statistical analysis were only collected from patients with a highest registered total IPC-time of at least 35 hours, which was used as the time-limit for the IPC-therapy to be considered per protocol.

Patients in the CTRL-group were given crutches and were treated with a standard below-knee plaster cast with the ankle in 30 degrees equinus position, with instructions of not bearing weight on the affected leg.

At the two week follow-visit, the initial post-operative treatment in both the IPC-group and the CTRL-group was replaced with a lower leg orthosis (Don-Joy Walker; DJO, Vista, California) to be used for another four weeks, during which the patients were instructed to apply full weight-bearing.

To cope with the postoperative pain, all patients were prescribed paracetamol $500 \mathrm{mg} /$ codeine $30 \mathrm{mg}$. No anti-inflammatory or thromboprophylactic agents were given to the patients during the post-operative treatment.

\section{Time to surgery (TTS)}

Patients were acutely planned for ATR surgery and operated on as soon as possible. However, since there are several injuries considered more urgent for surgery than ATR, TTS varied randomly between patients depending on the ratio of available hospital staff vs surgical work load. Neither the patient nor the surgeon could affect the time from ATR injury to surgery. Time to surgery (TTS), i.e. the time from ATR injury to start of the surgical procedure, was calculated by using the time point at which the patient sustained the injury as described in the patient journal, as well as the starting time point of the surgery as registered in the computerized operation report.

\section{Assessment of deep venous thrombosis (DVT)}

All patients were screened for DVT in the operated leg two weeks after ATR-surgery. The screening was performed by two experienced ultrasonographists, blinded to the post-operative treatment alternatives, using compression duplex ultrasound (CDU) performed with a Philips CX 50 ultrasound machine (Philips Medical Systems, Andover, MA, USA). In the process of screening for DVT, all deep proximal and distal veins, including muscle veins, as well as vena saphena magna were evaluated. The full criteria for DVT diagnosis and the diagnostic procedure have been described earlier (7). In short, thrombosis that involved the popliteal vein or any more proximal veins, with or without involvement of the calf veins was defined as a proximal DVT. An earlier study has shown that clinical diagnosis of DVTs in patients with rupture of the Achilles tendon is not possible, due to the similarity of its clinical features to normal post-operative findings, for this reason, there was no attempt to correlate scan findings with clinical features (3). Diagnosis of DVT was based on transversal ultrasound compression test of the blood vessel, and vein blood flow was assessed by color Doppler flow, two weeks after surgery. The second part of the screening was performed when the second ultrasonographists re-evaluated initial DVT-findings through analysis of CDU film sequences after completion of the study. This post boc examination verified the first diagnosis by demonstrating DVTs in the same anatomical localizations as the initial analysis.

\section{Statistical analysis}

Statistical analysis was performed to determine the significance levels of differences in DVT-incidence as well as odds ratios and $95 \%$ confidence intervals. Pearson's $\chi 2$-test was used for comparisons of categorical variables between groups (Table I-II) and subgroups (Table III-IV) and the independent samples T-test was used for comparisons of continuous variables (Table I and V). Logistic regression was used for the final subgroup-analysis (Figure 2), as well as to confirm that BMI and sex were not factors affecting each other to reduce the DVT-preventive effect of IPC among females and patients with low BMI. IPC-treatment, age and TTS were all significantly correlated to DVT-incidence in univariate Pearson correlation-analysis and thus age and TTS were corrected for in the final subgroup-analysis, examining the DVT-preventive effect of IPC (Figure 2). The level of significance was set to $<0.05$ (two-tailed). Among all the variables analyzed, only sex had a skewed distribution. For consistency, 95\% confidence interval was used for all variables when presenting the variance, including the skewed sex-variable. SPSS v24.0 software (SPSS Inc., Chicago, Illinois) was used for all analyses.

Groups were created based on patient characteristics:

1. Males and females were sorted into separate groups.

2.

a. The median value was calculated for each of the remaining patient characteristics (age, BMI and TTS), when looking at all patients (IPC and CTRL).

b. The median-value for each patient characteristic was rounded to the closest integer value. 
c. For each predictive factor, the corresponding median integer was used as a divider in both the IPC-groups and the CTRL-groups, creating subgroups roughly equal in size.

Using the steps described above, the following groups were created; male sex, female sex, age $<39$ years, age $\geq 39$ years, BMI <26, BMI $>26$, TTS $<66$ hours and TTS $>66$ hours.
These groups were then compared regarding DVT-incidence, initially examining the whole patient sample (plaster cast and IPC, Table II) and in the subsequent subgroup-analysis, within each treatment-alternative separately (CTRL or IPC, Table III-IV). In the final comparison, each subgroup in the CTRLgroup was compared to its corresponding subgroup within the IPC-group, as visualized in the forest plot (Figure 2).

Table II. Unadjusted comparisons of DVT incidence between groups

\begin{tabular}{|c|c|c|c|c|}
\hline \multirow{2}{*}{$\begin{array}{l}\text { Variable } \\
\text { Treatment }\end{array}$} & \multicolumn{2}{|c|}{$\%$ DVT } & \multirow[t]{2}{*}{ OR $(95 \% \mathrm{CI})$} & \multirow[t]{2}{*}{ p-value } \\
\hline & IPC & CTRL & & \\
\hline & $21.2 \%$ & $37.1 \%$ & $0.46(0.21-0.98)$ & 0.042 \\
\hline \multirow[t]{2}{*}{ Age, years } & $\geq 39$ & $<39$ & & \\
\hline & $55.3 \%$ & $15.6 \%$ & $6.06(2.44-15.06)$ & $<0.001$ \\
\hline \multirow[t]{2}{*}{ Sex } & Male & Female & & \\
\hline & $33.8 \%$ & $42.9 \%$ & $0.49(0.19-1.28)$ & 0.14 \\
\hline \multirow[t]{2}{*}{ BMI, $\mathrm{kg} / \mathrm{cm} 2$} & $>26$ & $<26$ & & \\
\hline & $26.4 \%$ & $32.8 \%$ & $0.74(0.35-1.55)$ & 0.42 \\
\hline \multirow[t]{2}{*}{ TTS, hours } & $>66$ & $<66$ & & \\
\hline & $38.2 \%$ & $21.2 \%$ & $2.30(1.07-4.95)$ & 0.031 \\
\hline
\end{tabular}

Abbreviations: DVT - Deep Venous Thrombosis, OR - Odds Ratio, CI - Confidence Interval, CTRL - Control group with immobilization in plaster cast, IPC - group with Intermittent Pneumatic Compression, BMI - Body Mass Index, TTS - Time to Surgery, P-value in bold $<0.05$.

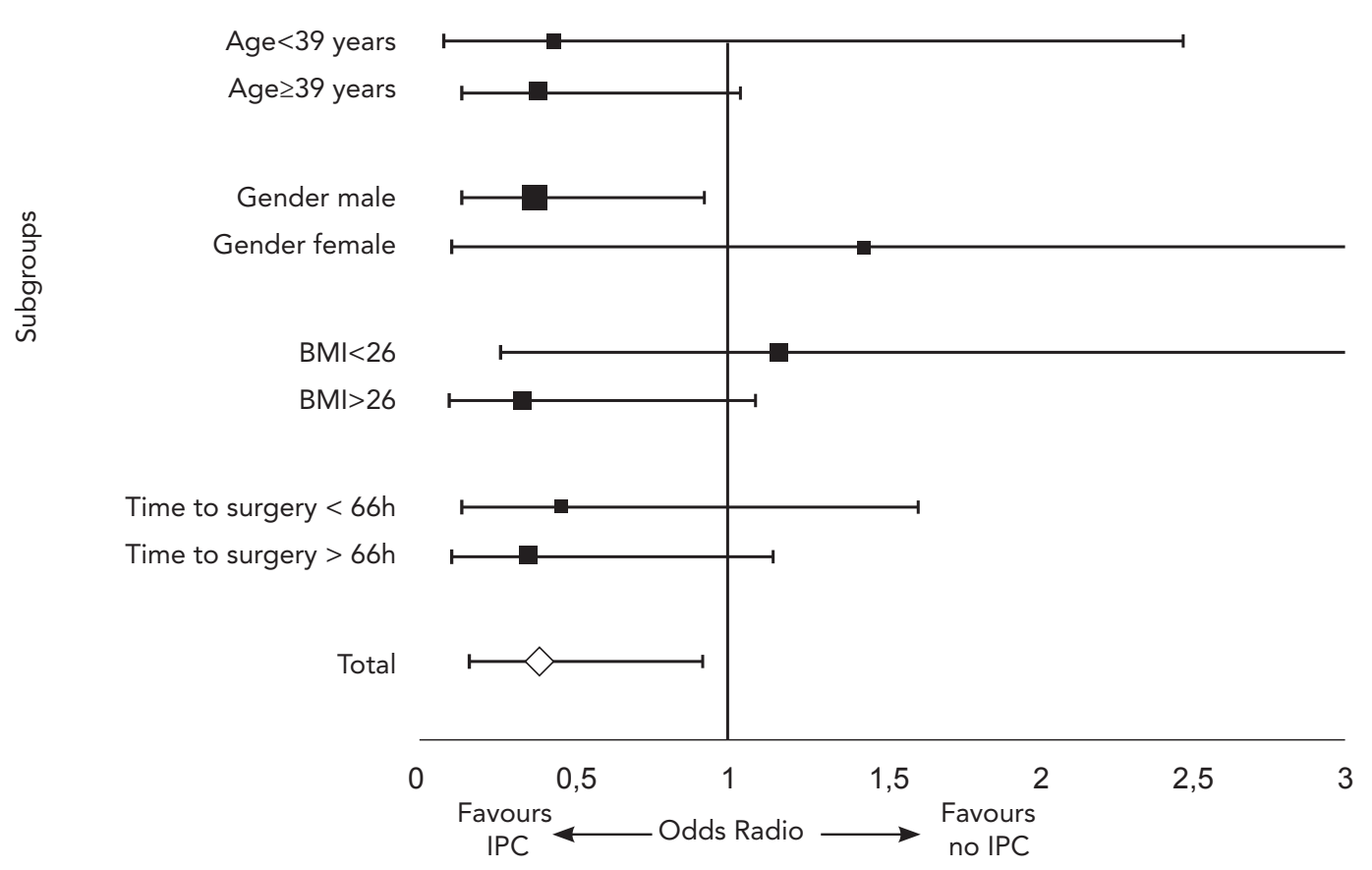

Figure 2. Forest plot depicting benefit of IPC-treatment vs CTRL in different subgroups. 
Table III. Incidence of DVT in subgroups allocated to plaster cast immobilization (CTRL)

\begin{tabular}{|c|c|c|c|c|}
\hline \multirow{2}{*}{$\begin{array}{l}\text { Variable } \\
\text { Age, years }\end{array}$} & \multicolumn{2}{|c|}{ \% DVT in CTRL subgroup } & \multirow[t]{2}{*}{ OR $(95 \% \mathrm{CI})$} & \multirow[t]{2}{*}{ p-value } \\
\hline & $\geq 39$ & $<39$ & & \\
\hline & $55.3 \%$ & $15.6 \%$ & $6.67(2.16-21.04)$ & 0.001 \\
\hline \multirow[t]{2}{*}{ Sex } & Female & Male & & \\
\hline & $46.2 \%$ & $35.1 \%$ & $1.59(0.47-5.36)$ & 0.46 \\
\hline \multirow[t]{2}{*}{ BMI, $\mathrm{kg} / \mathrm{cm} 2$} & $>26$ & $<26$ & & \\
\hline & $34.3 \%$ & $38.2 \%$ & $0.84(0.32-2.25)$ & 0.73 \\
\hline \multirow[t]{2}{*}{ TTS, hours } & $>66$ & $<66$ & & \\
\hline & $47.2 \%$ & $26.5 \%$ & $2.49(0.91-6.79)$ & 0.073 \\
\hline
\end{tabular}

Abbreviations: DVT - Deep Venous Thrombosis, CTRL - Control group with immobilization in plaster cast, OR - Odds Ratio, CI - Confidence Interval, BMI - Body Mass Index, TTS - Time to Surgery. P-value in bold $<0.05$

Table IV. Incidence of DVT in subgroups allocated to adjuvant IPC

\begin{tabular}{lcccc}
\hline Variable & \multicolumn{2}{c}{$\%$ DVT in IPC subgroups } & OR (95\% CI) & p-value \\
\hline Age, years & $\geq 39$ & $<39$ & & $\mathbf{0 . 0 1 2}$ \\
\hline Sex & $32.4 \%$ & $6.9 \%$ & & \\
\hline & Female & Male & & 0.23 \\
\hline BMI, $\mathrm{kg} / \mathrm{cm} 2$ & $37.5 \%$ & $19.0 \%$ & $2.56(0.53-12.38)$ & \\
\hline & $>26$ & $<26$ & $0.67(0.20-2.19)$ & 0.50 \\
\hline TTS, hours & $18.9 \%$ & $25.9 \%$ & & $2.11(0.62-7.20)$ \\
\hline
\end{tabular}

Abbreviations: DVT - Deep Venous Thrombosis, IPC - Intermittent Pneumatic Compression, OR - Odds Ratio, CI - Confidence Interval, BMI - Body Mass Index, TTS - Time to Surgery, P-value in bold $<0.05$

Table V - Comparison of IPC compliance between postoperative treatment groups.

\begin{tabular}{|c|c|c|c|}
\hline \multirow{2}{*}{$\begin{array}{l}\text { Variable } \\
\text { Age, years }\end{array}$} & \multicolumn{2}{|c|}{ IPC pump time, hh:mm, (95\%CI) } & \multirow[t]{2}{*}{ p-value } \\
\hline & $\geq 39$ & $<39$ & \\
\hline & 87:04 (74:53-99:15) & $89: 05(76: 16-101: 54)$ & 0.82 \\
\hline \multirow[t]{2}{*}{ Sex } & Female & Male & \\
\hline & $98: 45(52: 42-144: 47)$ & $86: 28(78: 06-94: 50)$ & 0.36 \\
\hline \multirow[t]{2}{*}{ BMI, $\mathrm{kg} / \mathrm{cm} 2$} & $>26$ & $<26$ & \\
\hline & $83: 42(71: 58-95: 25)$ & 93:35 (79:20-107:49) & 0.28 \\
\hline \multirow[t]{2}{*}{ TTS, hours } & $>66$ & $<66$ & \\
\hline & $87: 05(75: 44-98: 25)$ & $88: 49(74: 27-103: 10)$ & 0.85 \\
\hline
\end{tabular}

Abbreviations: IPC - Intermittent Pneumatic Compression, hh:mm - hours:minutes, CI - Confidence Interval, BMI - Body Mass Index, TTS - Time to Surgery. P-value calculated with t-test. 


\section{RESULTS}

\section{Factors affecting the risk of DVT}

When looking at all patients (CTRL and IPC), higher age (OR 6.06, p<0.001) and longer TTS (OR 2.3, p=0.031) significantly increased the risk of DVT, while IPC-treatment significantly reduced the risk of DVT (OR 0.46, $\mathrm{p}=$ 0.042) (Table II), with a relative risk reduction of $43 \%$. After correction for age and TTS, the overall benefit of IPC became even more prominent (OR $0.40, p=0.032$, Figure 2). Sex and BMI did not have any significant effect on the overall DVT-incidence (Table II).

\section{Subgroup risk of DVT during leg immobilization and adjuvant IPC treatment}

The specific subgroup risk of DVT during plaster cast immobilization, CTRL, and adjuvant treatment with IPC are presented in Table III and IV.

Age: The incidence of DVT in the CTRL-group was significantly higher among older patients compared to younger ( $55 \%$ vs. $16 \%, \mathrm{p}=0.001$ ). In the IPC-group the DVT-incidence was reduced among both older and younger patients ( $32 \%$ vs. $7 \%, \mathrm{p}=0.012)$, but the significant difference in DVT-incidence remained.

Sex: The risk of DVT was non-significantly higher among females both in the CTRL- (46\% vs. $35 \%, \mathrm{p}=0.46)$ and in the IPC-group ( $38 \%$ vs. $19 \%, \mathrm{p}=0.23$ ).

BMI: The DVT-incidence was comparable in patients with low- versus high BMI both in the CTRL- (38\% vs. $34 \%$, $\mathrm{p}=0.73$ ) and in the IPC-group ( $26 \%$ vs. $19 \%, \mathrm{p}=0.50)$.

TTS: The DVT-risk among CTRL patients exhibited a trend to be higher with long- compared to short TTS (47\% vs. $27 \% \mathrm{p}=0.073$ ), while among IPC patients the DVT-risk was non-significantly lower with short- vs. long TTS (16\% vs. $28 \%, \mathrm{p}=0.23$ ).

\section{WHICH SUBGROUP BENEFITS THE MOST FROM DVT-PREVENTION WITH IPC?}

In general, the DVT-preventive effects of adjuvant IPC were more prominent, with a lower OR and narrower confidence interval in the subgroups with the highest risk of DVT, as seen after correction for age and TTS (Figure 2).

Age: IPC exhibited a trend to reduce the risk of DVT from $55 \%$ to $32 \%$ among older patients (OR 0.39, p=0.059,

Figure 2). Among younger patients, there was a non-significant reduction in DVT-risk from $16 \%$ to $7 \%$ when using IPC (OR 0.44, $\mathrm{p}=0.35)$.
Sex: IPC significantly reduced the incidence of DVT from $35 \%$ to $19 \%$ among males (OR $0.35, \mathrm{p}=0.029$ ). Among females, however, the DVT-incidence decreased non-significantly from $46 \%$ to $38 \%$, but when corrected for age and TTS the OR was above 1 (OR 1.44, $\mathrm{p}=0.78$ ), implying that females might not have a benefit of using adjuvant IPC. BMI: Among patients with high BMI, IPC-treatment resulted in a DVT-reducing trend from $34 \%$ down to $19 \%$ (OR $0.33, \mathrm{p}=0.068$ ). Among patients with low BMI the DVT-risk decreased non-significantly from $38 \%$ to $26 \%$ and when corrected for age and TTS patients with low BMI seemed not to benefit from adjuvant IPC (OR 1.16, $\mathrm{p}=0.84$ ).

TTS: IPC non-significantly reduced the risk of DVT to a less extent in patients with short TTS, from $27 \%$ to $16 \%$ (OR $0.46, \mathrm{p}=0.22$ ), versus long TTS, from $47 \%$ to $28 \%$ (OR $0.35, \mathrm{p}=0.083)$.

\section{Compliance with IPC usage}

Among patients randomized to IPC-therapy, the number of hours the IPC-device was used, as assessed by both the patient and the device, did not correlate significantly to either age, sex, BMI or TTS ( $p>0.25$ ) (Table V). The self-registered application of IPC, $70.3 \mathrm{~h}$, correlated well with the device-registered usage of 67.3 hours.

\section{DISCUSSION}

This study demonstrated that ageing and longer waiting time to surgery increased the patient's risk of sustaining a DVT during leg immobilization after ATR-surgery, while post-operative treatment with IPC reduced the risk of DVT. Adjuvant IPC-therapy applied under an orthosis displayed a good DVT-preventive effect in most of the subgroups analyzed, whereas in females and patients with low BMI, however, the benefit of IPC seemed questionable.

The study established that most leg-immobilized patients exhibit around $40 \%$ relative risk reduction in the incidence of DVT while using adjuvant IPC. This DVT-preventive effect of IPC was also seen in the majority of the patient subgroups analyzed and is corroborated by conclusions of several Cochrane reviews of surgical patients receiving adjuvant IPC during bed immobilization $(8,9)$. The findings of this article seem to extend the DVT protective indications of IPC from inpatient care to become true also for care of many outpatients, especially for leg immobilized patients after an ATR.

The observation that the subgroups with the highest incidence of DVT in general exhibited the best effect of IPC, i.e. lowest odds ratio, suggests that IPC is more effective in 
patients with higher risk of DVT, as also suggested earlier (10). This conclusion was supported by the finding that the overall effects of IPC became stronger when corrected for age and TTS, two factors that significantly affected the risk of DVT.

The finding that age was the greatest risk factor, with an OR of 6, to sustain a DVT during leg-immobilization, regardless of postoperative treatment, should be accounted for when counselling patients and deciding post-operative treatments. Although the DVT-risk associated with increasing age has been shown before $(11,12)$, this is the first study to show how higher age among ATR-patients on its own affects the risk to sustain a DVT on a subgroup-level. The fact that our study is based on ATR-patients, with a large range (18-71) and variation in age (median 39), enables us to recognize DVT-risk differences associated to age that are not readily detected in studies with more age-homogenous patient bases. For example, similar studies researching stroke- or hip fracture patients, where the range and variation in age tend to be limited to higher ages, are seldom able to detect any statistically significant correlation between age and the risk of DVT (10).

Our findings showing that leg-immobilized patients already from around the age of 40 are of increased risk of suffering DVT suggest that these patients should receive DVT-prophylaxis. Presumably, IPC-therapy would be a good treatment option for DVT-prevention during leg-immobilization for patients over the age of 40 with the observed low OR for this patient cohort.

In this study we also discovered significantly higher incidence of DVT among patients with long- as compared to short TTS. Complications related to longer TTS are well documented, especially in disciplines such as hip fracture surgery (13-15).

The association between long TTS and higher risk of DVT may be explained by a longer waiting period, during which the muscle pump is inactivated. Patients sustaining an ATR are less likely to activate the muscle pump function in their affected limb. A longer period of time with sub-optimal blood flow creates a greater risk for formation of a clot in the deep veins of the immobilized limb.

The association between long TTS and increased DVT-incidence suggests means to decrease the time to surgery of ATR-patients and/or applying IPC during the waiting period, which was not the case in this study. The observation of a higher DVT-incidence in the CTRL-group compared to the IPC-group, may reflect that the DVT-risk with long TTS is minimized with the muscle pump-imitating function of the IPC-device applied after surgery. The observation moreover indicates that IPC in addition to a DVT-prophylactic function also exhibits DVT-resolving effects. This conclu- sion would be in line with the indication that patients with long TTS obtained better DVT-preventive effect of IPC than patients with short TTS.

In the subgroup analysis examining the DVT-preventive effect, we found that males and patients with high BMI exhibited a low OR in favor of IPC, while females and patients with low BMI exhibited a high OR not in favor of a DVT-preventive effect of IPC. This observation could be flawed by the fact that females in general exhibit lower BMI. However, when accounting for the effect of BMI and sex using logistic regression, the reduced effectiveness of IPC remained independent among both females and patients with low BMI.

The observed discrepancy in efficacy of IPC when it comes to females and patients with low BMI is novel and renders a closer look at potential underlying factors. The baseline DVT-incidence did not differ significantly between males and females or between patients with high and low BMI, and was therefore ruled out as a plausible reason for the discrepancy. IPC-compliance was also controlled for, but was found to be non-significantly different between all subgroups.

The fact that IPC demonstrated a clear DVT-preventive effect among males, but not among females, may instead be due to hormonal influences, a factor not controlled for in this study. Contraceptives and postmenopausal hormone therapy have been shown to be independent factors increasing the risk of DVT among females $(15,16)$. It may be possible that hormonal treatment also reduces the DVT-preventive effects of IPC. This conclusion, however, warrants further trials.

Women generally exhibit a lower calf muscle/adipose ratio and smaller calf circumference compared to men $(4,17)$. Less muscle mass may reduce the ability of the IPC-device to fully compress the blood out of the deep veins of the calf, which may a contributing factor reducing the DVT-preventive effect among women. However, this cannot be confirmed at this point, as there are currently no studies examining how either the sex or the muscle mass and circumference of the calf affects the efficiency of IPC.

The postoperative treatment alternatives of non-weight bearing immobilization in a plaster cast vs IPC under an orthosis, with weight bearing as tolerated, might have been a limitation of this study, since not only IPC was compared to the controls, but also the use of an orthosis with weight bearing. This setting was however chosen to be able to compare the intervention of IPC to the "standard of care" after ATR, i.e. non-weight bearing in a plaster cast, since the comparison to "standard of care" would not have been possible if the control-group also had been fitted with an orthosis with weight bearing. The alternative to apply the patients in the 
intervention-group with an IPC-device under a plaster cast was also considered, but was deemed too impractical to implement in a home-environment setting. Furthermore, the potential DVT-preventive effect of weight bearing in an orthosis was ruled out, since the difference in DVT-incidence between the groups had disappeared at the 6-week compression duplex ultrasound, following the period from two to six weeks postoperatively, where both groups were assigned to weight bearing in an orthosis without IPC. This is discussed further in the original article (5).

Despite observing an overall statistically significant DVT-reducing effect of IPC when looking at the whole patient sample, the most apparent limitation of this study was the reduction in statistical power encountered when creating subgroups, which rendered several subgroup-analyses statistically non-significant. Therefore, the findings from the subgroup-analyses should predominantly be used for discussion and a basis for future studies. Another limitation, which is common to most studies on ATR-patients, was the small number of females in relation to the number of males, which made conclusions on effects related to sex slightly uncertain. Another possible limitation was the retrospective nature of the study, with all the potential bias that follows. These limitations highlight the need for future RCTs with similar subgroup-analyses, to confidently apply the knowledge gained from the studies in a clinical setting.

\section{REFERENCES}

1. Bauersachs RM. Clinical presentation of deep vein thrombosis and pulmonary embolism. Best Pract Res Clin Haematol. 2012;25(3):243-51.

2. Arverud ED, Anundsson P, Hardell E, et al. Ageing, deep vein thrombosis and male gender predict poor outcome after acute Achilles tendon rupture. Bone Joint J. 2016;98-B(12):1635-41.

3. Lapidus LJ, Rosfors S, Ponzer S, et al. Prolonged thromboprophylaxis with dalteparin after surgical treatment of achilles tendon rupture: a randomized, placebo-controlled study. J Orthop Trauma. 2007;21(1):52-7.

4. Testroote M, Stigter WA, Janssen L, Janzing HM. Low molecular weight heparin for prevention of venous thromboembolism in patients with lower-leg immobilization. Cochrane Database Syst Rev. 2014(4):CD006681.

5. Domeij-Arverud E, Labruto F, Latifi A, Nilsson G, Edman G, Ackermann PW. Intermittent pneumatic compression reduces the risk of deep vein thrombosis during post-operative lower limb immobilisation: a prospective randomised trial of acute ruptures of the Achilles tendon. Bone Joint J. 2015;97$\mathrm{b}(5): 675-80$.

6. Padulo J., Oliva F., Frizziero A., Maffulli N. Basic principles and recommendations in clinical and field Science Research: 2018 update. MLTJ 2018; 8(3): 305 - 307.

7. Lapidus L, de Bri E, Ponzer S, Elvin A, Noren A, Rosfors S. High sensitivity with color duplex sonography in thrombo-
In order for such subgroup-analysis to provide statistically significant results that may be applied in clinical practice, a larger study-sample must be examined.

\section{CONCLUSIONS}

This is the first study examining the effect of IPC on different subgroups following lower-leg injury and immobilization. In this study we demonstrated that age over 38 and longer waiting time to surgery were significant risk-factors for DVT, and that IPC significantly reduced the risk of DVT. However, we also showed that the DVT-preventive effect may differ between various subgroups, and that subgroups with higher DVT-risk may have a greater benefit of IPC-treatment. We also discovered that there might be subgroups of patients, like females and patients with low BMI, that for undetermined reasons have limited or no effect of IPC, in the prevention of DVT. We consider these findings to be very interesting, but also conclude that corroborative subgroup-analyses on a larger patient-sample are needed to fully confirm and understand the differences seen between subgroups in this study.

\section{Conflict of Interest}

The authors declare that they have no conflict of interest.

sis screening after ankle fracture surgery. J Thromb Haemost. 2006;4(4):807-12.

8. Kakkos SK, Caprini JA, Geroulakos G, et al. Combined intermittent pneumatic leg compression and pharmacological prophylaxis for prevention of venous thromboembolism. Cochrane Database of Systematic Reviews [Internet]. 2016; (9). Available from: http://cochranelibrary-wiley.com/ doi/10.1002/14651858.CD005258.pub3/abstract.

9. Naccarato M, Chiodo GF, Dennis M, Sandercock PA. Physical methods for preventing deep vein thrombosis in stroke. Cochrane Database of Systematic Reviews [Internet]. 2010; (8). Available from: http://cochranelibrary-wiley.com/doi/10. 1002/14651858.CD001922.pub3/abstract.

10. Dennis M, Sandercock P, Reid J, Graham C, Forbes J, Murray G. Effectiveness of intermittent pneumatic compression in reduction of risk of deep vein thrombosis in patients who have had a stroke (CLOTS 3): a multicentre randomised controlled trial. Lancet. 2013;382(9891):516-24.

11. Soomro Q, Yousuf N, Bhutto AA, Abro HA, Memon AA. Venous thromboembolism (VTE): risk assessment in hospitalized patients. J Coll Physicians Surg Pak. 2014;24(7):455-8.

12. Shibuya N, Frost CH, Campbell JD, Davis ML, Jupiter DC. Incidence of acute deep vein thrombosis and pulmonary embolism in foot and ankle trauma: analysis of the National Trauma Data Bank. J Foot Ankle Surg. 2012;51(1):63-8. 
13. Madhu R, Kotnis R, Al-Mousawi A, et al. Outcome of surgery for reconstruction of fractures of the acetabulum. The time dependent effect of delay. J Bone Joint Surg Br. 2006;88(9):1197-203.

14. Pincus D, Ravi B, Wasserstein D, et al. Association Between Wait Time and 30-Day Mortality in Adults Undergoing Hip Fracture Surgery. Jama. 2017;318(20):1994-2003.

15. Simunovic N, Devereaux PJ, Sprague S, et al. Effect of early surgery after hip fracture on mortality and complications: systematic review and meta-analysis. Cmaj. 2010;182(15):1609-16.

16. Grady D, Wenger NK, Herrington D, et al. Postmenopausal hormone therapy increases risk for venous thromboembol- ic disease. The Heart and Estrogen/progestin Replacement Study. Ann Intern Med. 2000;132(9):689-96.

17. Bergendal A, Kieler H, Sundstrom A, Hirschberg AL, Kocoska-Maras L. Risk of venous thromboembolism associated with local and systemic use of hormone therapy in peri- and postmenopausal women and in relation to type and route of administration. Menopause. 2016;23(6):593-9.

18. Kubo A, Yoshimatsu T, Nishida Y. [Relation of the maximum calf circumference with albumin and body mass index in elderly cases of chronic hospitalization]. Nihon Ronen Igakkai Zasshi. 2009;46(3):239-43. 